\title{
Wavelet Transform Analysis on Image Compression using SPIHT
}

\author{
Vidya B. \\ PG Student \\ Dept of ECE \\ PDIT, Hosapete, India
}

\author{
Kishore M. \\ Asst.Prof \\ Dept of ECE \\ PDIT, Hosapete, India
}

\author{
Guruprasad H. M. \\ Professor \\ Dept of ECE \\ PDIT, Hosapete, India
}

\begin{abstract}
Addressing to high speed and low memory requirements the wavelet domain had made easier compression using SPIHT Set Partitioning in Hierarchical Tree (SPIHT) is quad tree structure. The proposed work intends to achieve higher rate in image compression. SPIHT works on Discrete Wavelet Transform, image is coded efficiently with few bits and originality of the source image is decoded exactly in the reconstructed image. Lifting Wavelet Transform gained quality in the decoding scheme and to save subband coding time. Scanning technique to decide threshold at every step of partitioning, to regain original features in the reconstructed image algorithm has given higher throughput compare other techniques. The results obtained has proved the algorithm is more efficient in data compression.
\end{abstract}

\section{General Terms}

Compression, Algorithm, encode, decode, coefficient, significance, image, Efficiency, Wavelet, compression Ratio.

\section{Keywords}

Discrete Cosine Transform (DCT), Discrete Wavelet Transform (DWT), Embedded Zero Tree wavelet (EZW), LWT, SPIHT.

\section{INTRODUCTION}

Today accessing the information in digital world is growing and there is need for efficient representation of information for storing, processing and transmission. Storage hardware are memory limited, some irrelevant data ignored to compress data. Compression is a data redundancy to store and transmit, is because of correlation property that exists between the pixels. Image compression, branch of data compression is technique of reducing data followed by coding image with fewer bits. Compression are Loss and Lossless types, based on quality of compressed image [7]. Present focus is on lossless compression to maintain quality in decoded image. Traditionally transform such as DFT, DCT, DST are used for this purpose and became complexity as signal size increases also have complex computations and time consuming. DCT based JPEG (Joint Picture Expert Group) compression standard gives desired compression at moderate bit rate but image quality is degraded at higher compression ratio due to artifacts and are overcome by wavelet transform. Wavelet transform provides multi resolution analysis, scalability and flexibility in performance.

Digital Wavelet transform (DWT) is supreme to DFT both in time-frequency because of 'localization property' of wavelets [3]. DWT based compression e.g. today JPEG 2000 is popular over Discrete Cosine Transform (e.g. JPEG), providing improved compression ratio. Wavelet transform coding decomposes input into low and high components and uses human perception features to achieve data compression. EZW
Embedded Zero Tree wavelet works on subband coding consist two steps- dominant and subordinate pass [5] to code significant locations effectively using statistical properties. Algorithm uses threshold and is updated by periodic iterations. Repeated scanning for threshold in EZW takes high time for encoding and decoding and causes Gibbs phenomenon. This hierarchical decomposition enables scalable transmission and decoding with mark able compression ratio. W.A Pearlman and Said rooted new algorithm for compression called SPIHT Set Partitioning In Hierarchical Trees derived from EZW [1] to achieve high ratio in compression. Research on SPIHT proved that it gives higher compression efficiency with reduced complexity, less operational time and improved quality in decoded image [4].

\section{RELATED WORK}

This paper motivated by [4] to decode original features of source image in reconstructed image and also describes how to achieve less computation time using simple characteristics of human perception capabilities [9] by signal modelling. Efficient encoding by set partition sub band hierarchy using pixels significance test for progressive transmission at low rate are proposed [1, 2]. In [5], work on EZW marked the slight performance difference with SPIHT in coding and transmission. Different approaches used for compression are studied [10]. Comparison on various transform on image as signal processing are analyzed [6,7]. Tensor analysis of nature of image are rooted [8] and choice of wavelet transform direct the work to achieve desired compression .The work carried is to get desired CR, store in limited space and lower transmission delay, easy computation. All these are achieved by the algorithm presented here.

\section{PROBLEM STATEMENT}

There is scope for information running on digital world which includes audio, video and fields like robotics, medical, surveillance, education department etc. requires effective way of representing data to process and store for future work, compression technique made this possible considering higher de-correlation. Traditional real valued DCT works by dividing natural image in set of frequency components, low frequencies are efficiently exploited and high frequencies are quantized coarsely [8]. Quantization as step in image encoding induces error, results in loss of information and poor reconstructed image and has close approximation to Karhunen-Loeve KL transforms. KL is optimal, has it need ready Kernel for processing and is image dependency. All these are succeeded by the wavelet theory.

The problems associated in present Fourier based compression technique are listed here [8]: 
- Truncation results in blurring leads to lossy compression. - Gibbs Effect.

- Coarse quantization of spectral components induces graininess on image.

- Each block is independently encoded, blocking artifacts at the block boundaries has become serious.

- Higher encoding and decoding time because each block segment has to be computed independently.

Drawbacks of Fourier transforms are succeeded by wavelet transform. Discrete wavelet transform is powerful spectral and spatial analysis. The proposed work mainly concentrates on SPIHT algorithm to maintain quality in reconstruction, saves time required to encode and decode.

\section{METHODOLOGY}

The work presented completes in two divisions i.e. firstly a four level wavelet decomposition resulting non-overlapping bands after every step of partition of image data giving Lower Lower LL, Higher Lower HL, Lower Higher LH, and Higher Higher HH [3,6,9]. Then Decomposition is followed by sorting and refinement pass of SPIHT. Paper presents the use of two wavelet transform based on nature of image block using structure tensor analysis, which roots to decide choice of wavelet transform. Lifting wavelet transform (LWT) and Discrete wavelet transform (DWT) are used for comparative analysis of compression.

\subsection{Discrete Wavelet Transform}

The transform has desired special properties for high resolution analysis and suitable for image compression. Wavelet transform represents the signal in to different scaled components give time and frequency details used as frame work for signal processing using filter banks. Image decomposition using DWT by filtering leads two set of frequencies, higher ones containing detailed (wavelet function) part of signal and lowers corresponding to smooth variations (scaling factor). Discrete input signal $x_{0}, x_{1}, \ldots \ldots \ldots x_{m}$ are transformed in to wavelet domain by converting it into higher and lower coefficient sets [6].

$$
\begin{aligned}
& H_{i}=\sum_{m=0}^{k-1} x_{2 i-m} \cdot s_{m}(z) \\
& L_{i}=\sum_{m=0}^{k-1} x_{2 i-m} \cdot t_{m}(z)
\end{aligned}
$$

Where $S_{m}(z)$ and $t_{m}(z)$ are wavelet filters of length $k$ and $\mathrm{I}$ is 0 to $[n / 2]-1$. Decomposition in horizontal and vertical direction, resulting in splitting of image in to quad subbands i.e. LL, HL, LH and HH. The first filter level decomposition of signal is shown in figure 1 . This method is effective of deeper resolution analysis is controlled by threshold and details of the image below threshold are neglected.

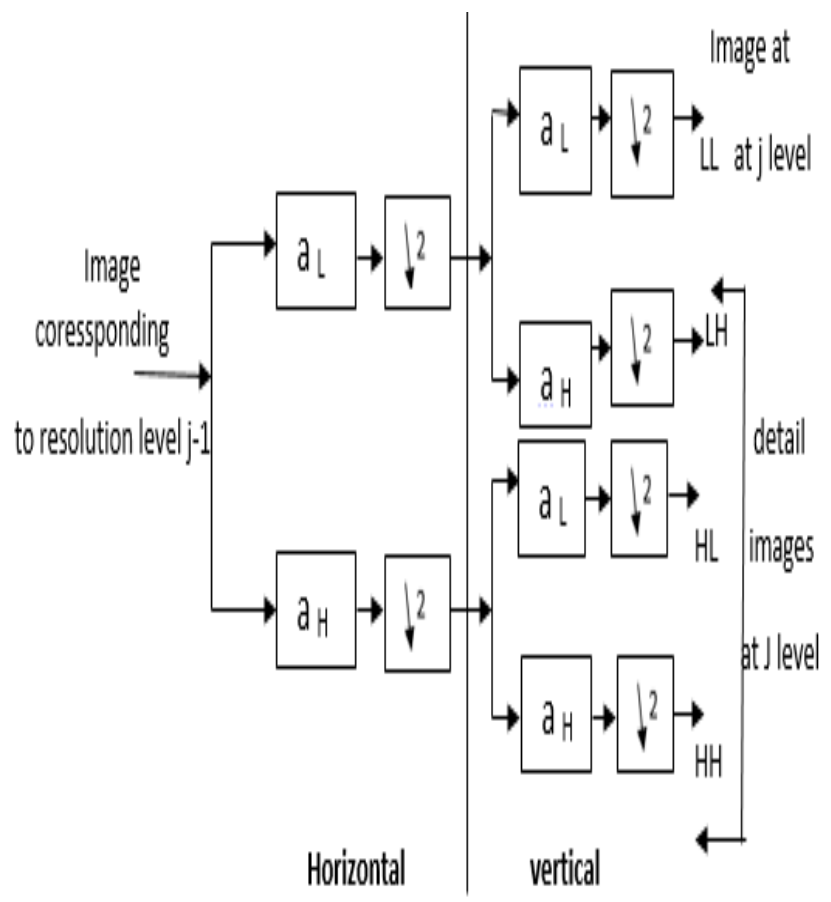

Fig 1.Filter level decomposition

After first step there are four bands and for each succeeding level, LL band of previous step acts as input to next level resulting in $\mathrm{LL}_{2}, \mathrm{HL}_{2}, \mathrm{LH}_{2}$, \& $\mathrm{HH}_{2}$ [6]. Next $\mathrm{LL}_{2}$ is decomposed and continued for number of levels to clear approximation for multiresolution. Three step wavelet decomposition is shown in figure. 2 .
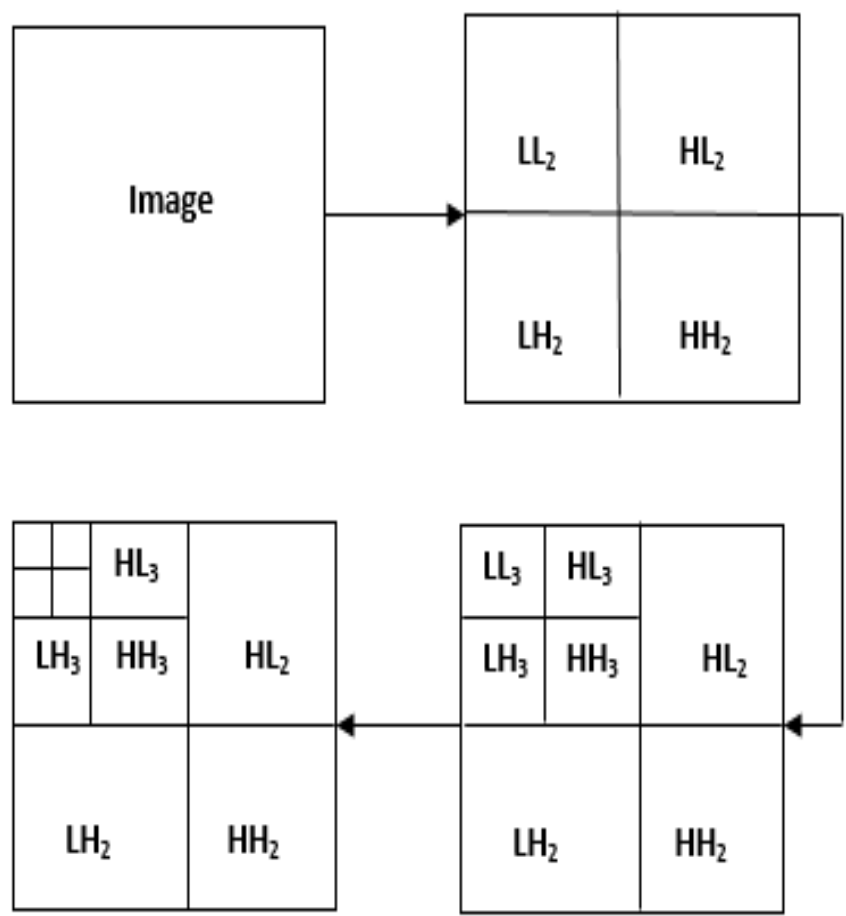

Fig 2. Three level Wavelet decomposition

Implementation of DWT is convolution filtering and complexity increases as length of filter increases. 


\subsection{Lifting Wavelet Transform}

LWT is best algorithm to compute wavelet transform compared to classic wavelet, it designs desired wavelet and performs DWT with lesser coefficients and reduces complexity with faster computation. Involves 3 steps [6].

1. Split: Input signal is divided in odd and even samples.

2. Predict: uses predict $P$ (.) operator based on original signal to estimate missing data from previous step and determines odd sequence using even coefficients. (Dual step lifting associated with HP filter)

3. Update: updates the even samples by replacing even set by averages computed by difference between odd set and even predictor previously stored.

Figure 3 shows forward Lifting wavelet transform. Lifting scheme wavelets are $2^{\text {nd }}$ generation wavelets which replaced need of translation and dilation factors. Other wavelet transform can be analyzed using this scheme with reduced complexity [6].

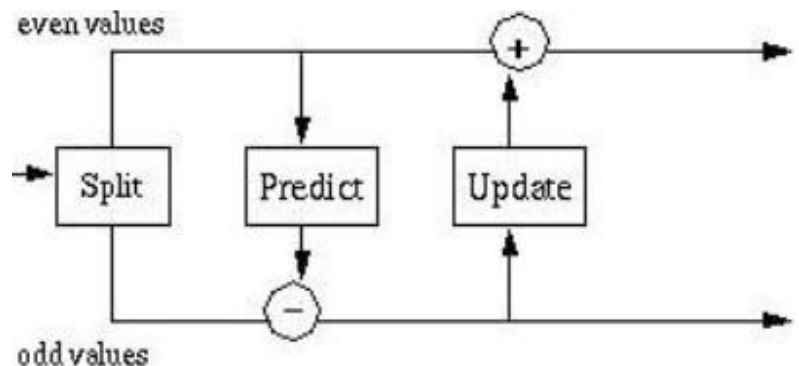

Fig 3. Forward Lifting wavelet Transform.

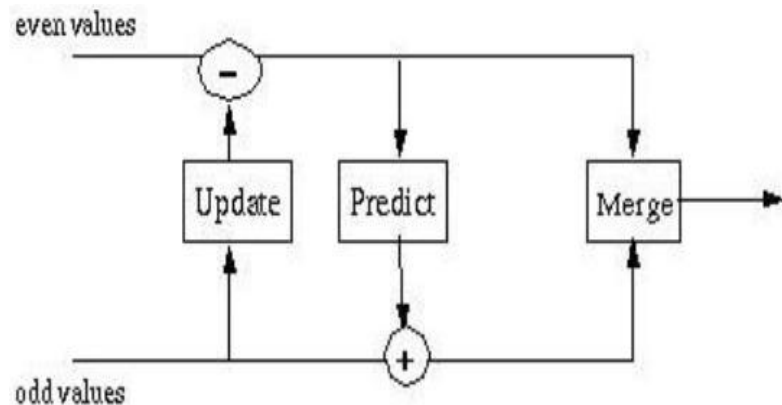

Fig 4. Reverse Lifting wavelet Transform.

The DWT needs series of filter banks for division of signal whereas LWT divides in zipper manner for cumulative convolution operation.

\section{PROPOSED WORK}

\subsection{Description of SPIHT}

SPIHT algorithm uses hierarchical rules partition so that most significant part are coded first for faster coding. Exploits some important features such as easy and simple, self-adaptive, efficient coding, embedded, idem potency and supports precise bit rate. It relates parental child relationship between wavelet coefficients for progressive transmission by identifying significant pixel. The categorization of coefficients in SPIHT as quad structure is shown in figure 5 and is called Spatial Orientation Tree (SOT).

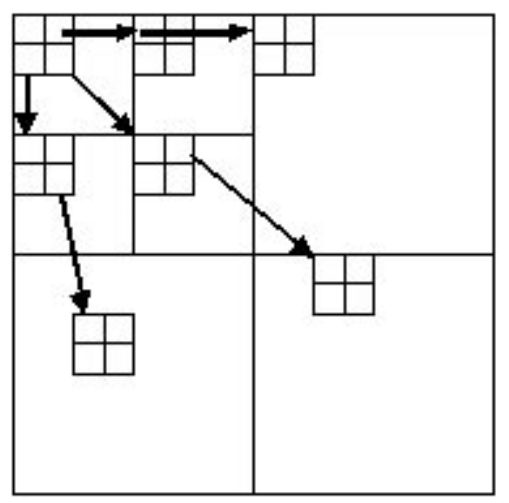

Fig.5. SOT of coefficients in SPIHT

Mainly involves two steps: Sorting pass and Refinement pass and are operated by threshold which keeps changing. The operational chart of algorithm is shown in figure 6 .

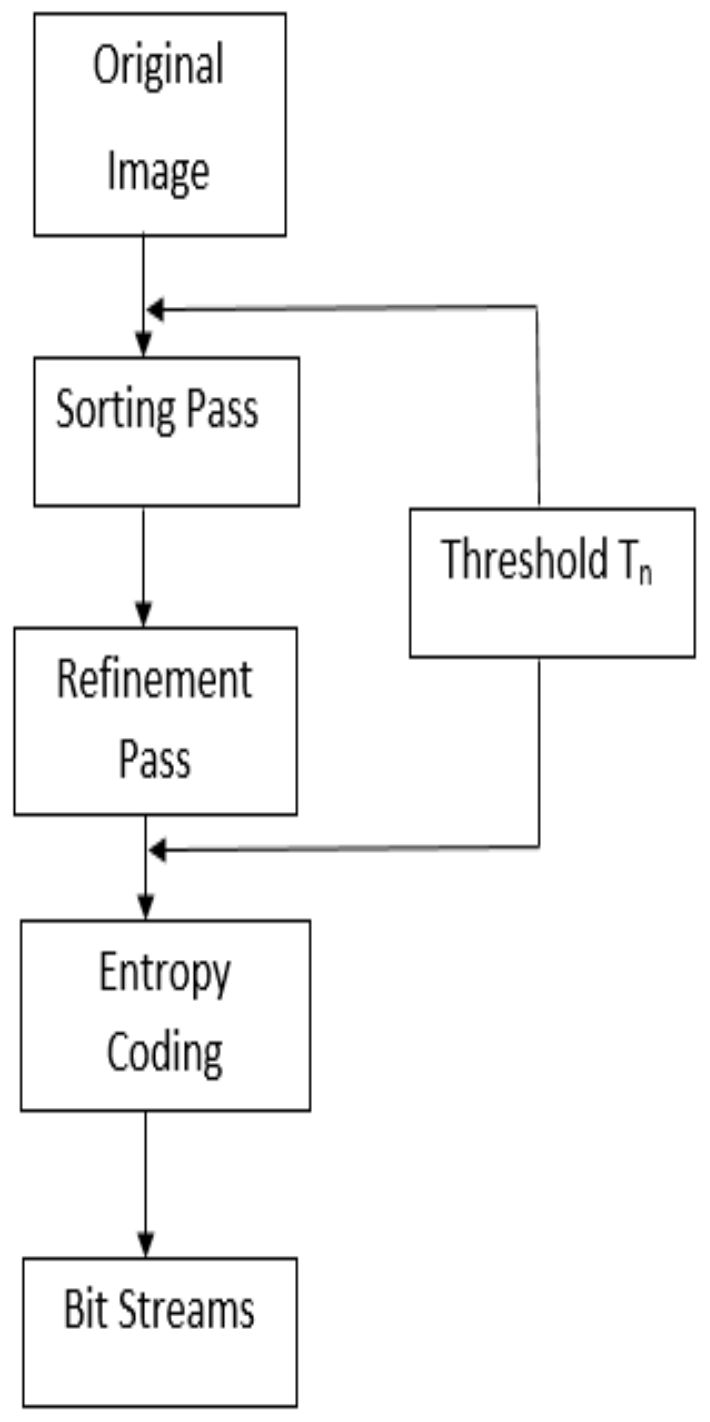

Fig 6. SPIHT flowchart

Entropy (energy contained) intends to average image length by coding symbols by probabilistic conditions. Entropy coding such as Huffman, Arithmetic coding are used. Algorithm supports different wavelet transforms. LWT and 
DWT are used here for compression using Spatial Orientation tree analysis. The three list of pixels categorized for coefficient distinguish. Term Coefficient is usual term for 'pixel' as analysis carried on transformed image. DWT is well supported for homogeneous image blocks and LWT for hetero images. For a given node i.e. pixel $\left(\mathrm{n}_{1} \mathrm{n}_{2}\right)$ or $(\mathrm{n})$ we define [3]-

$\mathrm{O}\left(\mathrm{n}_{1}, \mathrm{n}_{2}\right)$ : contains offspring's of $\left(\mathrm{n}_{1}, \mathrm{n}_{2}\right)$,

$\mathrm{D}\left(\mathrm{n}_{1}, \mathrm{n}_{2}\right)$ : descendent set has all subsequent nodes of $\left(\mathrm{n}_{1}, \mathrm{n}_{2}\right)$,

$\left.\mathrm{L} \mathrm{n}_{1}, \mathrm{n}_{2}\right)$ : contains difference of $\mathrm{D}\left(\mathrm{n}_{1}, \mathrm{n}_{2}\right)$ and $\mathrm{O}\left(\mathrm{n}_{1}, \mathrm{n}_{2}\right)$, given by $\mathrm{L}\left(\mathrm{n}_{1}, \mathrm{n}_{2}\right)=\mathrm{D}\left(\mathrm{n}_{1}, \mathrm{n}_{2}\right)-\mathrm{O}\left(\mathrm{n}_{1}, \mathrm{n}_{2}\right)$, except $\left(\mathrm{n}_{1}, \mathrm{n}_{2}\right)$ and

$\mathrm{H}$ contains all tree roots.

SPIHT explicit significant, insignificant pixels by test defined as

$$
\mathrm{S}(\mathrm{n} 1, \mathrm{n} 2)=\left\{\begin{array}{l}
1 ; \max (\mathrm{n} 1, \mathrm{n} 2) \in \mathrm{T}\{\mathrm{C}(\mathrm{n} 1, \mathrm{n} 2)\} \geq 2^{\mathrm{n}} \\
0 ; \text { otherwise. }
\end{array}\right.
$$

Threshold $\mathrm{T}=2^{\mathrm{n}} \cdot$ Lists categorized for coding are:

List of Insignificant sets LIS;

List of Insignificant Pixels LIP;

List of Significant Pixels LSP;

\section{Algorithm Steps:}

\section{Initialization}

Compute $\mathrm{n}=\left[\log 2\left(\max C\left(\mathrm{n}_{1}, \mathrm{n}_{2}\right)\right] ; \mathrm{C}(\mathrm{n} 1, \mathrm{n} 2) \max \right.$ coefficient.

$\mathrm{LSP}=$ null

LIP has roots of $\mathrm{H}$

LIS contains $\mathrm{D}\left(\mathrm{n}_{1}, \mathrm{n}_{2}\right)$ roots.

2. Sorting pass: (significance map coding)

a. LIP process: For each $(\mathrm{n} 1, \mathrm{n} 2)$ in LIP perform $\mathrm{S}(\mathrm{n})$, found 1 as significance output (0- insignificance) add to LSP with output sign $0 / 1=+/$ - and end LIP.

b. LIS process: For each $(\mathrm{n} 1, \mathrm{n} 2)$ in LIS find $\mathrm{S}(\mathrm{n})$ for coefficients if $S(n)=1$ then reject it and add to LSP else send to LIP.

If $\mathrm{L}(\mathrm{n} 1, \mathrm{n} 2) \neq 0$, then perform partitioning knowing it is descendant D set or L set and update according to list of pixels using $\mathrm{S}(\mathrm{n})$ test.

3. Refinement pass: Each coefficient in LSP except those added in previous step, encode the nth Most Significant Bit of C (n).

4. Quantization step: This step is followed by decreasing $n$ by one and repeat sorting.

Likewise coefficients of every set are sorted, refined and transmitted. Threshold of every subband keeps changing and scanning is important .Performance of image compression is evaluated by Peak Signal to Noise Ratio PSNR and Mean Square Error MSE. These two are inversely related by following equation;

$$
\mathrm{PSNR}=20 \log _{10} \frac{255}{\mathrm{MSE}}
$$

Noise is the error present in reconstructed image. For good compression, higher the value of PSNR lower is the MSE.

The compression rate or ratio $\mathrm{CR}$ is given by
Compression ratio $=$ Bit rate of compressed image.

$$
\text { Bit rate of input image. }
$$

The evaluation of SPIHT algorithm is explained by taking image as an example in next section.

\subsection{Working Example}

Consider $\mathrm{N} \times \mathrm{N}$ image e.g. $4 \times 4$ to analysis encoding of image using SPIHT.

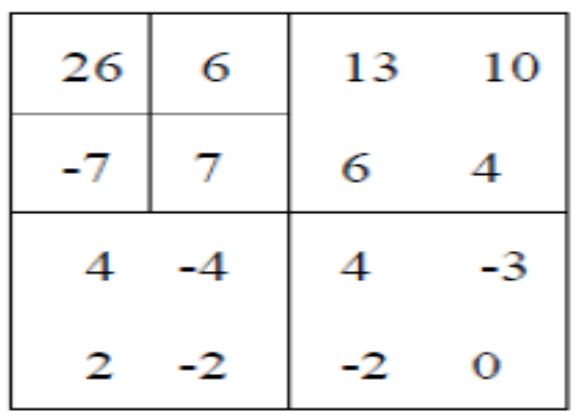

The following image is subjected to wavelet decomposition, each value indicating pixel coefficient in transform domain Calculate threshold for LL band using max coefficient i.e. 26.

$\mathrm{n}=\log 2 \max .($ Coefficient) $=4.7$, initial $\mathrm{T} 1=16$ implies 26>threshold load 26 to LSP. Initially LSP is null. Pixel sets are as

$\mathrm{LIP}=[26,6,-7,7]$ and

$\mathrm{LIS}=[13,10,6,4,4,-4,2,-2,4,-3,-2,0]$.

$\mathrm{LSP}=[\mathrm{empty}]$.

After first pass, second sorting threshold $\mathrm{T} 2=8$ and pixel 13 $\& 10$ satisfies condition needed and are loaded to LSP.

At second stage output steam is $(1110101110111010)_{b}$ and encoding completes in three level $\mathrm{T}_{3}=4$ with all significant coefficients from LIP and LIS loaded to LSP. Therefore after third sorting pass the coefficients are shifted to sets as:

$\mathrm{LSP}=[26,13,10,6,-7,7,6,4,-4,4,4]$;

$\mathrm{LIP}=[2,-2,-3,-2,0]$ and

Final LIS is empty with all significant coefficients loaded to LSP and output encoded bit stream is follows: $111010111011101011100111111111101100110001001100_{\mathrm{b}}$

\section{SIMULATION RESULTS}

The results obtained are executed in Matlab environment. Comparison of two methods proposed here are tabulated below. Observation details that improved compression ratio (CR) are obtained using SPIHT algorithm by DWT \& LWT. Compression performance is measured by PSNR and MSE, stating higher compression is achieved with high value of PSNR. The results obtained for few images are tabulated in Table 1. For each image both LWT and DWT are performed and results are validated below. Algorithm supports for both gray scale image and color images. Lifting wavelet transform gives higher compression than the discrete wavelet transform DWT. Here the performance of SPIHT are analyzed using two wavelet transforms. Results also shows that the algorithm has faster results by less time consumption. The wavelet decomposition of the Saturn image is indicated in fig 8 stating the LL band at high level decomposition gives more information than other bands. 


\section{SORTING PASS:}

Initialize: All Roots

Initialize: null

Initialize: D's roots

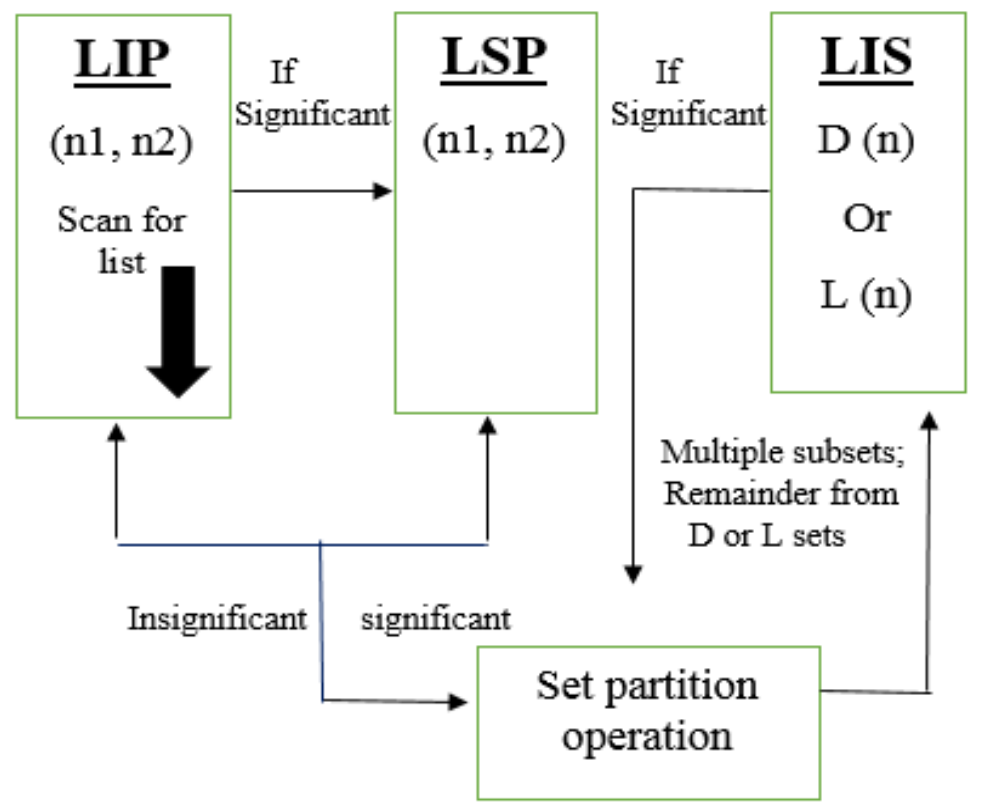

\section{REFINEMENT PASS}

Initialize: Empty

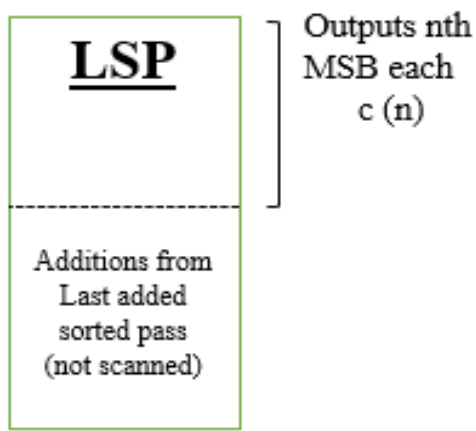

Fig 7. Operation of SPIHT

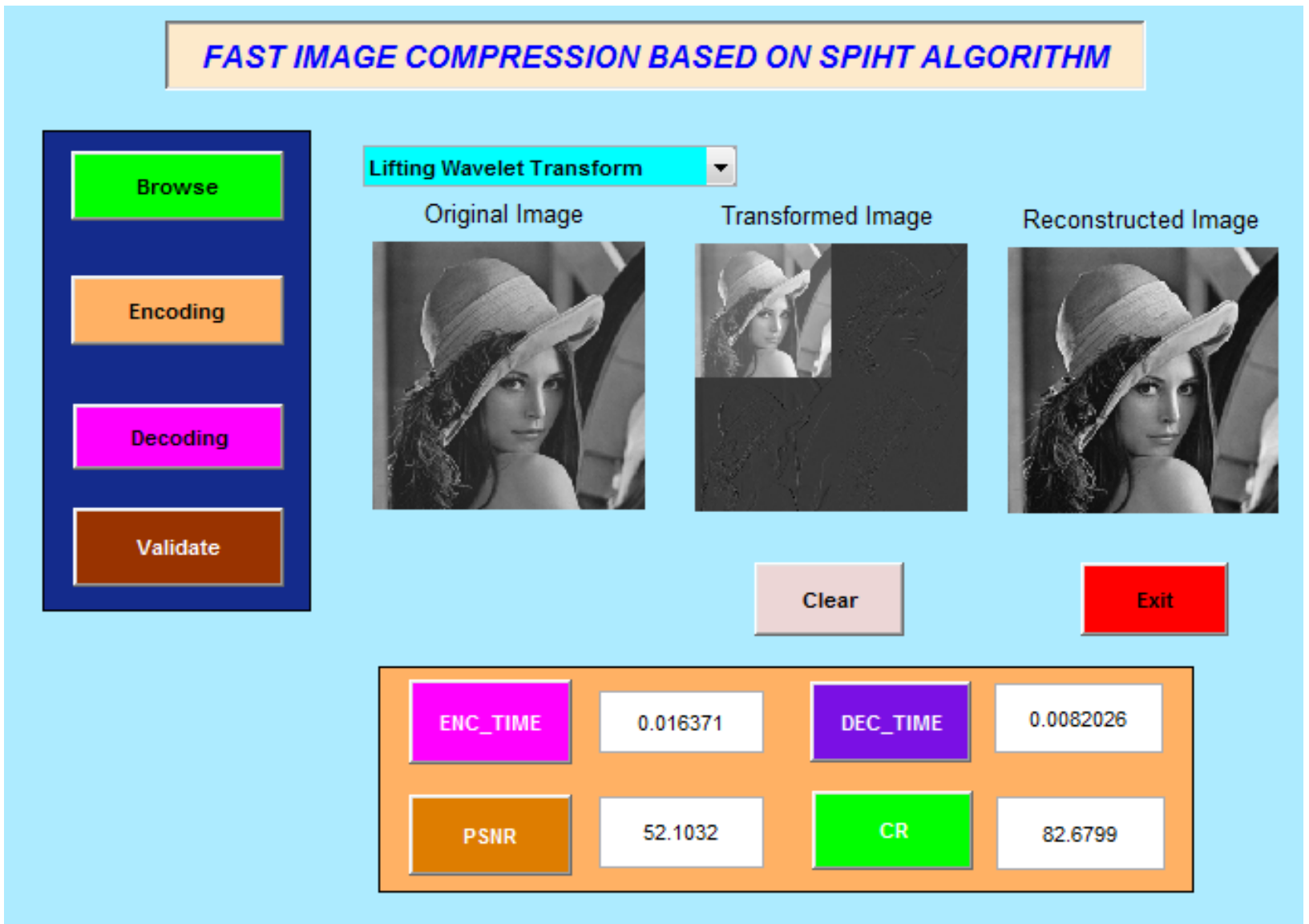

Fig.8. View of parameters recoding of compression 


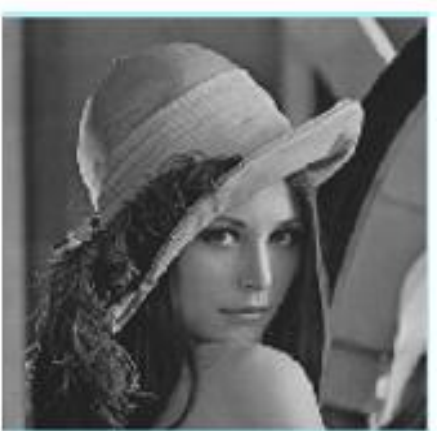

Fig.9 (a) Original Image

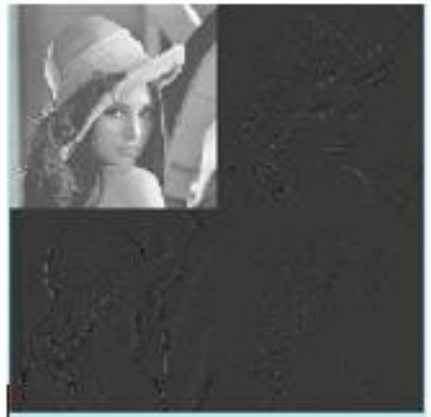

(b) Transformed image

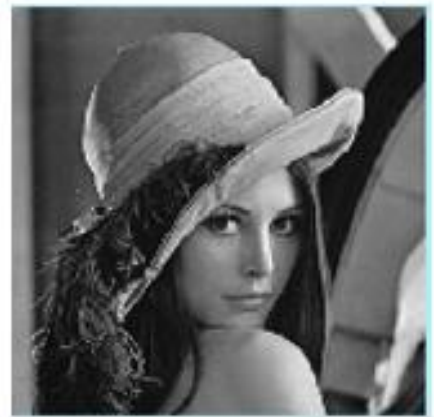

(c) Reconstructed image

Table 1. Stimulated Results

\begin{tabular}{|c|c|c|c|c|}
\hline Transform & \multicolumn{2}{|c|}{ Lifting scheme } & \multicolumn{2}{c|}{ DWT } \\
\hline \multirow{2}{*}{ Image 256x256 } & PSNR & CR & PSNR & CR \\
& $\mathrm{dB}$ & $\%$ & $\mathrm{~dB}$ & $\%$ \\
\hline Lena & $\mathbf{5 7 . 4 7 3 2}$ & $\mathbf{8 1 . 3 5 5}$ & $\mathbf{5 2 . 1 2 2 0}$ & $\mathbf{8 0 . 5 1 0}$ \\
\hline Gold hill & $\mathbf{5 7 . 2 0 1 5}$ & $\mathbf{8 5 . 6 0 7}$ & $\mathbf{5 2 . 0 4 7 6}$ & $\mathbf{8 4 . 8 4 1}$ \\
\hline Rice & $\mathbf{5 8 . 1 4 0 7}$ & 74.974 & $\mathbf{5 3 . 3 0 2 0}$ & 73.816 \\
\hline Moon & $\mathbf{6 1 . 3 0 4 0}$ & $\mathbf{6 3 . 9 2 2}$ & $\mathbf{5 6 . 5 5 7 4}$ & 63.758 \\
\hline Brain MRI & $\mathbf{5 7 . 2 0 7 4}$ & 78.810 & $\mathbf{5 2 . 6 0 2 0}$ & 78.251 \\
\hline
\end{tabular}

\section{CONCLUSION AND FUTURE SCOPE}

SPIHT algorithm has improved compression performance using wavelet transform to achieve high Compression Ratio. Lifting scheme speed up the operation and saves coding decoding time with reduced computation. DWT results to give multi resolution analysis. Decoder replicate the reverse execution to provide better reconstructed image. Features of Human visual system used for scanning threshold changes, provided best decoded image and less calculation. Results shows SPIHT is simple fast compression method for progressive transmission at variable bitrates. Because of numerous advantages this algorithm is used in wide range of applications.

Current methods of compression are quite away from desired ultimate goals. So there is a scope of optimal solution in coming era by challenging issues such as accurate image modelling, representation, computation, fast transmission and complexity have become present challenges for huge data compression in this digital world.

\section{REFERENCES}

[1] A.Said, and W.A.Pearlman, A New, Fast and Efficient Image Codec Based on Set Partitioning in Hierarchical Trees, IEEE Trans on Circ and Syst for Video Tech, vol.6, no.3, June 1996, pp.243-250

[2] Jia Zhi Gang, Guo Xiao Dong, Li Lin Sheng, A Fast Image Compression Algorithm Based on SPIHT, IEEE
Conference on Industrial Electronics and Applications 2009, pp. 3779-3781.

[3] A. Mallaiah, S.K. Shabbir, T. Subhashini, "An Spiht Algorithm with Huffman Encoder for Image Compression and Quality Improvement Using Retinex Algorithm” IJSTR vol.1, Issuse 5, June 2012.

[4] Ping Liu, Guanfeng Li, "An Improved SPIHT Algorithm for Image Compression in low bit Rate", Communication and Networks,2013, 4, 245-248.

[5] Shilpa Jaiswal, R.R Sedamkar "Performance Evaluation on EZW and SPIHT image compression techniques." International Journal of scientific and Research publications, Volume 4, issues 10, October 2014 ISSN 2250-3153.

[6] Renu Rani, Savita, and Sunita Virmani "Image Compression Using DCT, HAAR, and Biorthogonal Wavelets, LWT: A Comparative Analysis" International Journal of Engineering Trends and Technology IJETT, Volume4, Issuse- june 2013

[7] Saad AI-Azawi, Said Boussakta and Alex Yakovelv "Image Compression Algorithm Using Intensity Based Adaptive Quantisation Technique", American j. of Engg.and Applied Science 4(4): 504-512, 2011, ISSN 1941-7020. 
[8] Mrs. Keshika Jandge, Mr. Rohit Raja 'Image Compression Based on Discrete Wavelet and Lifting Wavelet Transform Technique" International Journal of Science, Engineering and Technology Research (IJSETR), Volume 3, issues 3, March 2014.

[9] N. J ayant, J. Johnston , and R Safranek, "Signal Compression based on models of Human perception ," Proc IEEE, vol 81, PP.138591422, oct 1993.

[10] Cebrail taskin, Serdar Kurat "An Overview of Image Compression Approaches". IEEE, Third International conference on Digital telecommunication, 2008.
[11] A. R. Calderbank, I. Daubechies, W. Sweldens, and B.L.Yeo, Lossless Iimage Compression Using Integer to Integer Wavelet Transforms, in Proc. IEEE Int. Conf. Image Processing, vol. 1, Santa Barbara, CA, Oct. 1997, pp. 596-599.

[12] Safranek and Johnston, A perceptually tuned sub-band quantization data compression, in proc. ICASSP 1989:1945-1948 